\title{
ТЕОРЕТИЧЕСКИЕ АСПЕКТЫ ЭМОЦИОНАЛЬНОГО ВЫГОРАНИЯ ПЕДАГОГИЧЕСКИХ РАБОТНИКОВ В ЗАРУБЕЖНЫХ ИССЛЕДОВАНИЯХ
}

\author{
Дзюбина Наталья Геннадьевна \\ магистрант 2 курса \\ направления 37.04.01 «Пихология» \\ профиль «Клиническая психология» \\ Кафедра «Психофизиология \\ и клиническая психология» \\ факультет «Психология, педагогика \\ и дефектология», ДГТУ
}

\begin{abstract}
Аннотация: В статье рассматривается эмоциональное выгорание (ЭВ) как один из факторов, влияющих на качество и производительность труда педагога, его здоровье и эффективность взаимодействия с субъектами образовательного процесса. Во введении автором раскрывается актуальность исследуемой темы в связи с проблемой стресса и эмоционального выгорания педагогов в образовательном пространстве. В теоретическом обосновании автором приводится обзор современных зарубежных исследований к рассмотрению феномена эмоционального выгорания у педагогов; влияния эмоционального выгорания на качество и производительность труда учителя. Новизна исследования заключается в выделении и систематизации научных знаний о проблеме эмоционального выгорания, описании и структурировании формально-содержательных характеристик феномена ЭВ. В разделе «Обсуждение результатов» автором выделяются основные направления исследования зарубежных авторов, которые позволяют говорить о важности исследования ЭВ в профессиональной деятельности педагогов. Автором подчеркивается недостаточная изученность связи ЭВ с когнитивными особенностями субъекта труда, намечаются дальнейшие перспективы исследования.
\end{abstract}

Ключевые слова: Эмоциональное выгорание, стресс, депрессия, ресурсы, профессиональная деятельность. 


\title{
THEORETICAL ASPECTS OF EMOTIONAL BURNOUT OF PEDAGOGICAL WORKERS IN FOREIGN STUDIES
}

\begin{abstract}
The article emotional burnout (EB) as one of the factors affecting the quality and productivity of a teacher's work, his health and the effectiveness of interaction with the subjects of the educational process. In the introduction, the author reveals the relevance of the topic under study in connection with the problem of stress and EB of teachers in the educational space. In the theoretical substantiation, the author provides an overview of modern foreign studies to consider the phenomenon of emotional burnout among teachers; the impact of emotional burnout on the quality and productivity of teacher work. The novelty of the research lies in the allocation and systematization of scientific knowledge about the problem of emotional burnout, description and structuring of the formal-meaningful characteristics of the EB phenomenon. In the section "Discussion of the results" the author highlights the main areas of research by foreign authors, which allow us to speak about the importance of the study of EB in the professional activities of teachers. The author emphasizes the insufficient study of the relationship between EB and the cognitive characteristics of the subject of labor, and outlines further research prospects.
\end{abstract}

Key words: Emotional burnout, stress, depression, resources, professional activity.

\section{ВВЕДЕНИЕ}

Педагогическая профессия характеризуется разнообразием задач при сложных условиях труда, в которых выделяются потенциальными факторы стресса: высокие требования к работе и психосоциальные взаимодействия с учениками, родителями и коллегами. По сравнению со специалистами других профессиональных групп педагоги больше подвержены стрессу на работе, тревоге, утомляемости и проблемам со сном. Кроме того, существует повышенный риск психических и психических расстройств, связанных со стрессом, психосоматические заболевания и выгорание $[8,15]$.

В Международной классификации болезней (МКБ-11) выгорание не рассматривается как болезнь, но в смысле явления, связанного с работой, в результате неудачного преодоление хронического стресса, связанного с работой [30]. Процесс выгорания типичен: после начальных фаз перегрузки психосоматические расстройства могут перерасти в физические и 
психологическое истощение и депрессию [3]. Одно из наиболее часто цитируемых определений синдрома выгорания восходит к Maslach and Jackson $[17,18]$. В современных зарубежных исследованиях рассматривается вопрос не только о внешних факторах, влияющих на формирование ЭВ, но и демографических, индивидуальных, психологических и личностных особенностях, составляющих специфический профиль личности с синдромом ЭВ и ее склонность к выгоранию.

Таким образом, цель исследования - рассмотреть проблему эмоционального выгорания педагогических работников в зарубежных исследованиях и выделить характеристики, уровни и профили эмоционального выгорания.

\section{ТЕОРЕТИЧЕСКОЕ ОБОСНОВАНИЕ}

Исследование влияния специфики труда на самочувствие специалистов все чаще, в последние годы, привлекает внимание ученых. Кроме того, изменение условий труда и жизнедеятельности вызвало увеличение подверженности специалиста психосоциальному риску, а частота негативных факторов оказывает влияние на развитие у субъекта труда психосоматических и профессиональных болезней [8].

Junne et al. пишут, что увеличение психосоциальных рисков вызывает увеличение стресса, связанных с работой [12]. В настоящее время связанные с профессиональной деятельностью стресс - одно из основных расстройств здоровья на рабочем месте и основной барьер для профессионального развития. Кроме того, несколько исследований показали, что стресс, связанный с работой снижает социальное взаимодействие, вызывает трудности с концентрацией внимания на работе, вызывает физиологическую боль и сердечно-сосудистые проблемы, усиливает психические заболевания в виде депрессий и беспокойства [19]. Кроме того, стресс, связанный с работой, связан с увеличением текучести кадров, нарушениями в профессиональной деятельности и выполнении обязанностей, снижении производительности труда, увеличении несчастных случаев на производстве [19].

Последствием стресса на работе является синдром выгорания. Выгорание было определено Maslach и др. как психологический синдром в ответ хроническим межличностным и эмоциональным стрессорам на работе. Характерные воздействия можно наблюдать у работников сферы услуг, педагогов, полицейских, медицинских работников и т.д. [25]. Всемирная организация здравоохранения вслед за Международным Классификация 
болезней (МКБ) 11, определяет выгорание как результат хронического стресса на рабочем месте, с которым сотрудники не справляются [30]. Выгорание связано с различными последствиями, такие как невыход на работу, проблемы со здоровьем, увеличение ошибок за смену, снижение производительности и эффективности труда работник и депрессии $[2,3,9,17,18,27]$.

Зарубежные исследователи отмечают, что распространенность выгорания в образовании колеблется между 11 и 35,5\%, в зависимости от страны и исследования считается [9]. В Испании несколько исследований показали, что 48\% педагогов в системе образования имеют высокий уровень выгорания и 52\% профессионалов в области образования имеют средний уровень выгорания [24].

Некоторые исследования связали эти уровни выгорания с эмоциональным истощением [4], а также эмоциональным интеллектом [22]. Кроме того, самоэффективность и ресурсы выживания играют важную роль в управление такими факторами, как перегрузка, неоднозначность ролей и конфликты, давление со стороны (администрации, родителей и т.п.) на педагогов, недостаточность ресурсов (психологических, эмоциональных, социальных, экономических и т.п.), плохие условия труда, отсутствие профессионального признания, низкий уровень вознаграждения, неучастие в принятии решений, неэффективное общение, конфликты между персоналом и плохое поведение воспитанников [4].

Учитывая эту распространенность в последние годы, исследование выгорания в сфере образования становится все более важным. Большинство исследований указывало на важность изучения выгорания педагогов (, считая это риском для специалиста и фактором негативного влияния на их благополучие (физическое и психологическое), эффективность педагогической деятельности, взаимодействия с воспитанниками, их мотивации на работе, прогулы, депрессии, бессонницы, или снижение способности оказывать поддержку обучающимся $[5,14,21]$.

Несколько исследований были сосредоточены на изучении конкретного эмоционального выгорания и профиля педагогической деятельности [21].

В психологии существует несколько моделей ЭВ. В модели Karasek отражается влияние профессиональных требований, ресурсов и последствий психосоциальных рисков на выгорание специалиста[13]. Характеристики выгорания рассматриваются в модели Maslach and Jackson, в модели GilMonte. Maslach and Jackson объяснили, выгорание формированием трех 
показателей: эмоциональное истощение, обезличивание и снижение личных достижений [17, 18]. Gil-Monte добавил к этой трехмерной модели новое измерение - чувство вины $[9,18,23]$. В этой модели выгорание понимается как эмоциональное и когнитивное ухудшение, которое вызывает негативное отношение и поведение по отношению к клиентам или пользователям организации как стратегия выживания[9]. На основе этой модели Gil-Monte разработал шкалу эмоционального выгорания (SBI) для оценки уровня выгорания по 4 измерениям: энтузиазм на работе, психосоциальное истощение, праздность и чувство вины. Энтузиазм по отношению к работе определяется как желание испытуемого исполнять задачи и достигать цели на работе, потому что это источник личных удовольствие. Психологическое истощение определяется как появление эмоций и физического истощения на работе, потому что сотрудникам необходимо ежедневно решать проблемы клиентов, воспитанников, учеников. Праздность, по мнению Gil-Monte, - это проявление негативного безразличия и цинизма по отношению к воспитанникам[9]. Еще проявляется вина, которая вызвана отрицательным отношением к коллегам, воспитанникам, развивалась в процессе труда. Сочетание этих компонентов позволяют идентифицировать два профиля выгорания и уровни тяжести выгорания у профессионалов [9].

Профиль 1 включает субъектов, которые сообщают о более высоких уровнях психосоциального истощения, циничного поведения, т. е. праздности, и когнитивного ухудшения, но не высокое чувство вины. Этот профиль описывает сотрудников, которые страдают от стресса и дискомфорта на работе. В этом профиле циничное отношение - т. е. праздность - используются как стратегия выживания, и это позволяет учителю контролировать уровень стресса и его последствий.

Профиль 2 включает сотрудников с более высоким уровнем психосоциального истощения, циничного поведения, когнитивного ухудшения и высокого уровня чувства вины. Эти сотрудники испытывают более серьезный дискомфорт на работе [9]. Им помогают справиться стратегии, основанные на циничном отношении, но они не так эффективны, как в профиле 1. Кроме того, циничное отношение и праздность в рабочей среде как стратегия выживания воспринимается сотрудниками, клиентами, воспитанниками как неадекватная [7].

Наряду с этими профилями выгорания можно рассматривать два других уровня выгорания. С одной стороны, есть люди, не страдающие ЭВ (люди с 
высоким уровнем энтузиазм по отношению к работе и низкий психологическим уровнем истощения и лености). Но с другой стороны, есть люди, которые не страдают выгоранием, но близки к тому, чтобы страдать от этого (рабочие с более низким уровнем энтузиазм по отношению к работе и более высоким психологическим уровнем истощения, низким уровнем праздности и вины), что приводит к выше среднего уровню выгорания.

Guidetti et al. нашел несколько профилей выгорания, соответствующих этим уровням. Существование этих уровней и профилей является ключевыми, учитывая, что каждый из профиля требуют различных схем вмешательства [7], а также разные уровни риска и последствий для специалиста [10]. Несмотря на проблемы, Guidetti et al., в своем исследовании, не анализировали, какие переменные влияют на проявление типов уровня выгорания, не выявлено какие прогнозируемые переменные оказывают воздействие на переход с одного уровня на другой. Авторы отмечают, что исследование выгорания являются важной и очевидной проблемой, как и необходимость исследовать уровни выгорания. Это позволяет понять и классифицировать сотрудников, сосредоточить внимание на стратегиях выживания, которые используют сотрудники и ресурсов, необходимых в профессиональной деятельности [7].

Хотя различные исследования подчеркивают важность того, что в появлении выгорания могут быть разные факторы, нет исследований анализирующих, какие факторы определяют большую вероятность принадлежности к тому или иному уровню. Модель Karasek имеет самую фундаментальную теоретическую и эмпирическую поддержку, которая в настоящее время имеет в науке наибольшее влияние и внимание. Это модель объясняет стресс, связанный с работой, дисбалансом между психологическими требованиями на работе (например, рабочая нагрузка, межличностные конфликты, дисбаланс, неоднозначность ролей и конфликт ролей) и уровнем контроля или ресурсов, которыми располагает сотрудник (например, автономия, обратная связь и т. д.) [13].

Johnson and Hall добавили переменную социальная поддержка как третье измерение контроля. В соответствии с этой моделью есть зависимость между здоровьем, благополучием сотрудника, балансом рабочих требований и личных ресурсов специалиста. Когда требования выше, чем ресурсы, тем сильнее ощущение стресса на работе. Кроме того, хронический стресс, связанный с работой, может вызвать синдром выгорания. Синдром может 
проявляться в виде нескольких расстройств здоровья или психосоматических симптомов [11].

Следующие требования выделяются своей важностью: межличностные конфликты, нагрузка, дисбаланс, конфликт ролей и неоднозначность ролей. Эти переменные положительно коррелируют с выгоранием. Более того, в последние годы среди прочих возник еще один фактор - эмоциональный труд. Skaalvik and Skaalvik в своем исследовании среди учителей, показали, что конфликтное взаимодействие с родителями и учениками вызывает наибольшее напряжение у учителей $[20,26,31]$. Исследование показало отрицательную связь между напряжением, самоэффективностью и ее проявлением в психологическом истощении и обезличивании. С другой стороны, Gonçalves et al. нашел взаимосвязь между выгоранием и рабочей нагрузкой. Эта переменная была предиктором эмоционального истощения[6]. Unda в своем исследовании обнаружила отрицательную связь с энтузиазмом по отношению к работе, положительным отношением с эмоциональным истощением, чувством вины и праздностью[28]. Кроме того, Хи в своем исследовании педагогов обнаружил связь между ролевым конфликтом, эмоциональным истощением и обезличиванием. Автор отметил, что конфликт ролей будет уменьшать энтузиазм и энергию для работы. Также Chang and Chu в своем исследовании обнаружили связь между ролевой неоднозначностью и эмоциональным истощением, а также негативную связь с личным достижением [25].

Как говорилось выше, в последние годы эмоциональный труд привлек внимание многих исследователей. Несколько исследований показали, что взаимосвязь между эмоциональным трудом и выгоранием [1, 20, 31]. Yilmaz et al. выявили, что у учителей естественные эмоции и поверхностные актерская игра предсказывала выгорание, эмоциональное истощение, и обезличивание [31]. Кроме того, подавление эмоций и эмоциональный диссонанс был связан с выгоранием [1]. Более того, Pisaniello et al. нашли положительную взаимосвязь между поверхностным действием, эмоциональным истощением, обезличиванием и отрицательным отношением к личному достижение [20].

Wegge et al. в своем исследовании объяснили положительную связь между эмоциональным диссонансом, эмоциональным истощением, обезличиванием и негативную связь с личным достижением [29]. Переменные ресурсов отрицательно связаны с выгоранием. В качестве переменных ресурса выделены факторы: автономия работы, социальная поддержка и ресурсы в 
профессии. Некоторые переменные, такие как социальная поддержка, рассматриваются авторами как фактор защиты от выгорания [6].

\section{ОБСУЖДЕНИЕ РЕЗУЛЬТАТОВ}

Проведенный анализ позволяет выделить следующие направления исследований, по которым можно судить о важности проблемы эмоционального выгорания как для субъекта образования, так и для качества и эффективности образовательной. Первое направление связано с изучением влияния стресса и условий труда на развитие ЭВ. Второе направление исследует последствия ЭВ на качество и производительность труда субъектов образования. Третье направление сосредоточило внимания на влияние демографических и личностных характеристик на склонность к формированию ЭВ у специалистов.

Кроме того, установлены факторы, оказывающие влияние на формирование синдрома ЭВ:

1) превышение требований приведет к отрицательным последствиям для сотрудников: более высокое выгорание. Однако наличие достаточных ресурсов приносит пользу сотруднику, уменьшая вероятность иметь эмоциональное выгорание и истощение[24];

2) некоторые социально-демографические переменные могут быть связаны с выгоранием. Например, возраст и уровень образования. Пол - это переменная, которая широко изучается в связи с выгоранием [15, 16]. Обнаружены различные исследования в выборках учителей что у женщин уровень выгорания выше, чем у мужчин. Кроме того, исследование метаанализа показало, что женщины чаще сообщают о выгорании, чем мужчины. Также, некоторые исследования показали, что возраст имеет положительную связь с эмоциональным истощением, то есть с возрастом нарастает выгорание. Однако все меняется, когда сотрудники становятся среднего возраста и уровень выгорания снижается [15]. Разные исследования уровня образования и ЭВ показали различные результаты. Некоторые исследования не обнаружили разницы между уровнями образования, а другие исследования показали более высокий уровень выгорание в средней школе [16]. Важность исследования синдрома выгорания широко доказана, особенно у педагогов.

3) однако не было обнаружено исследований, подтверждающих влияния когнитивных особенностей педагогов на развитие ЭВ. 


\section{ЗАКЛЮЧЕНИЕ}

На сегодняшний день эмоциональное выгорание педагогов является одной из серьезных проблем в педагогике и психологии. Психологическое здоровье, а значит эмоциональное благополучие позитивно сказывается на субъектах образовательной системе и качестве труда учителя и воспитателя. Противоположный эффект мы можем наблюдать, если у педагога проявляются симптомы ЭВ в профессии. Это приводит к срывам занятий, нарушению отношений между субъектами учебно-образовательной деятельности, болезням и истощению педагогов.

В результате проведенного анализа зарубежной литературы по данной проблеме были сделаны следующие выводы.

1. ЭВ как психологический синдром, возникающим в ответ на хронические межличностные и эмоциональные стрессоры на работе.

2. Выделяют несколько уровней ЭВ и 2 профиля.

3. Основными факторами, способствующие развитию ЭВ являются личностное отношение к работе и ресурсы профессионала, условия труда, уровень образования, пол, возраст.

Тема ЭВ в науке еще недостаточно изучена, она требует дальнейшего рассмотрения и более углубленного анализа с точки зрения когнитивной психологии. К примеру, еще не выявлены когнитивные качества личности, определяющие склонность к эмоциональному выгоранию у педагогов. Дальнейшие исследования, в том числе эмпирического характера, помогут уточнить выдвинутые нами предположения о влиянии когнитивных особенностей педагогов на развитие ЭВ, выделить функции эмоциональных и когнитивных свойств личности в структуре ЭВ, более полно исследовать данный феномен. Автор заявляет об отсутствии конфликта интересов.

\section{Список литературы}

1. Andela, M., Truchot, D., and Borteyrou, X. (2015). Emotional labour and burnout: some methodological consideration and refinements. Can. J. Behav. Sci. 47, 321-332. doi: 10.1037/cbs0000024

2. Bakker, A. B., and Demerouti, E. (2017). Job demands-resources theory: taking stock and looking forward. J. Occup. Health Psychol. 22, 273-285. doi: 10.1037/ocp0000056 
3. Bakker, A. B., and Wang, Y. (2020). Self-undermining behavior at work: evidence of construct and predictive validity. Int. J. Stress Manag. 27, 241-251. doi: $10.1037 /$ str0000150

4. Betoret, F. D. (2009). Self-efficacy, school resources, job stressors and burnout among Spanish primary and secondary school teachers: a structural equation approach. Edu. Psychol. 29, 45-68. doi: 10.1080/01443410802459234

5. Cecho, R., Švihrová, V., Cecho, D., Novák, M., and Hudecková, H. (2019). Exposure to mental load and psychosocial risks in kindergarten teachers. Zdr Varst. 58, 120-128. doi: 10.2478/sjph-2019-0016

6. Gonçalves, A., Fontes, L., Simães, C., and Gomes, A. R. (2019). "Stress and burnout in health professionals," in Occupational and Environmental Safety and Health, eds P. Arezes et al. (Cham: Springer), 563-571.

7. Guidetti, G., Viotti, S., Gil-Monte, P. R., and Converso, D. (2018). Feeling guilty or not guilty. Identifying burnout profiles among Italian teachers. Curr. Psychol. 37, 769-780. doi: 10.1007/s12144-016-9556-6

8. Fornell, B., Correa, M., del Amo, M. P. L., and Martín, J. J. (2018). influence of changes in the Spanish labor market during the economic crisis (20072011) on perceived health. Qual. Life Res. 27, 2095-2105. doi: 10.1007/s11136-018$1824-5$

9. Gil-Monte, P.R. (2012). Influence of guilt on the relationship between burnout and depression. Eur. Psychol. 17, 231-236. doi: 10.1027/1016-9040/a00 0096

10. Jin, Y. Y., Noh, H., Shin, H., and Lee, S. M. (2015). A typology of burnout among Korean teachers. Asia Pac. Educ. Res. 24, 309-318. doi: 10.1007/s40299-014$0181-6$

11. Johnson, J. V., and Hall, B. M. (1988). Job strain, work place social support and cardiovascular disease: a cross sectional study of a random sample of the Swedish working population. Am. J. Public Health 78 1338-1342. doi: 10.2105/AJPH.78.10.1336

12. Junne, F., Michaelis, M., Rothermund, E., Stuber, F., Gündel, H., Zipfel, S., et al. (2018). The role of work-related factors in the development of psychological distress and associated mental disorders: differential views of human resource managers, occupational physicians, primary care physicians and psychotherapists in Germany. Int. J. Environ. Res. Public Health 15, 559-576. doi: 10.3390/ ijerph15030559 
13. Karasek, R. A. (1979). Job demands, job decision latitude, and mental strain: implications for job redesign. Adm. Sci. Q. 24, 285-308. doi: $10.2307 / 2392498$

14. Kim, L.E., and Buric, I. (2019). Teacher self-efficacy and burnout: determining the 'directions of prediction through an autoregressive cross-lagged panel model. J. Educ. Psychol. 11, 1661-1676. doi: 10.1037/edu0000424

15. LaFaver, K., Miyasaki, J. M., Keran, C. M., Rheaume, C., Gulya, L., Levin, K. H., et al. (2018). Age and sex differences in burnout, career satisfaction, and well-being in US neurologists. Neurology 91, e1928-e1941. doi: 10.1212/WNL.0000000000006497

16. Langher, V., Caputo, A., and Ricci, M. E. (2017). The potential role of perceived support for reduction of special education teachers' burnout. Int. J. Educ. Psychol. 6, 120-147. doi: 10.17583/ijep.2017.2126

17. Maslach, C., Schaufeliu, W. B., and Leiter, M. P. (2001). Job burnout. Ann. Rev. Psychol. 52, 397-422. doi: 10.1146/annurev.psych.52.1.397

18. Maslach, C. (2017). Finding solutions to the problem of burnout. Consul. Psychol. J. Pract. Res. 69, 143-152. doi: 10.1037/cpb0000090

19. Nielsen, K., Jørgensen, M. B., Milczarek, M., and Munar, L. (2018). Healthy Workers, Thriving Companies: A Practical Guide to Well-Being at Work. Luxembourg: European Agency for Safety and Health at Work.

20. Pisaniello, S. L., Winefield, H. R., and Delfabbro, P. H. (2012). The influence of emotional labour and emotional work on the occupational health and well-being of South Australian hospital nurses. J. Vocat. Behav. 80, 579-591. doi: 10.1016/j.jvb.2012.01.015

21. Pyhältö, K., Pietarinen, J., Haverinen, K., Tikkanen, L., and Soini, T. (2021). Teacher burnout profiles and proactive strategies. Euro. J. Psychol. Educ. 36, 219-242. doi: 10.1007/s10212-020-00465-6

22. Puertas-Molero, P., Zurita-Ortega, F., Chacón-Cuberos, R., MartínezMartínez, A., Castro-Sánchez, M., and González-Valero, G. (2018). An explanatory model of emotional intelligence and its association with stress, burnout syndrome, and non-verbal communication in the university teachers. J. Clin. Med. 7:524. doi: 10.3390/jcm7120524

23. Rabasa, B., Figueiredo-Ferraz, H., Gil-Monte, P. R., and Llorca-Pellicer, M. (2016). The role of guilt in the relationship between teacher's job burnout syndrome and the inclination toward absenteeism. Rev. Psicodidáct. 21, 103-119. doi: 10.1387/RevPsicodidact.13076 
24. Ruiz-Calzado, I. (2016). Burnout en docentes de educación especial de Córdoba (españa). Opción 32, 569-588. Salmela-Aro, K., Hietajärvi, L., and Lonka, K. (2019). Work burnout and engagement profiles among teachers. Front. Psychol. 10:2254. doi: 10.3389/fpsyg.2019.02254

25. Sarisik, M., Bogan, E., Zengin, B., and Dedeoglu, B. B. (2019). The impact of burnout on organizational commitment: a study of public sector employees in Turkey. J. Glob. Busin. Insights 4, 106-118. doi: 10.5038/2640-6489.4.2.1066

26. Skaalvik, E., and Skaalvik, S. (2007). Dimensions of teacher self-efficacy and relations with strain factors, perceived collective teacher efficacy, and teacher burnout. J. Educ. Psychol. 99, 611-625. doi: 10.1037/0022-0663.99.3.611

27. Stehman, C. R., Testo, Z., and Gershaw, R. S. (2019). Burnout, drop out, suicide: physician loss in emergency medicine, part I. West. J. Emerg. Med. 20, 485494. doi: 10.5811/westjem.2019.4.40970

28. Unda, S. (2010). Estudio de prevalencia del síndrome de quemarse por el trabajo (SQT) y su asociación con sobrecarga y autoeficacia en maestros de primaria de la Ciudad de México. Cienc Trab 35, 257-262.

29. Wegge, J., Van Dick, R., and von Bernstorff, C. (2010). Emotional dissonance in call centre work. J. Manage. Psychol. 25, 596-619. doi: $10.1108 / 02683941011056950$

30. World Health Organization (2019). International Classification of Disease. Ginevra: World Health Organization.

31. Yilmaz, K., Altinkurt, Y., Guner, M., and Sen, B. (2015). The relationship between teachers' emotional labor and burnout level. Euras. J. Educ. Res. 59, 75-90. doi: 10.14689/ejer.2015.59.5

(C) Н.Г. Дзюбина, 2021 